\title{
Parents' Awareness and Oral Health Care Measures of Pediatric Patients Receiving Chemotherapy
}

\author{
Shahad S Alkhuwaiter ${ }^{1}$ \\ 'Department of Orthodontic and Pediatric Dentistry, College of \\ Dentistry, Qassim University, Qassim, Saudi Arabia
}

\begin{abstract}
Address for correspondence: Shahad S Alkhuwaiter, Department of Orthodontic and Pediatric Dentistry, College of Dentistry, Qassim University, Qassim Region, Buraydah 52571, Saudi Arabia

E-mail: shahd.alkhuwaiter@gmail.com
\end{abstract}

\begin{abstract}
Objective: The purpose of this study was to assess the parents' awareness and practices towards oral health care of children receiving chemotherapy.

Materials and Methods: Data was collected through a questionnaire-based survey at King Abdullah Specialized Children Hospital (KASCH), Department of Pediatric Hematology and Oncology. The questionnaire consisted of 15 questions regarding the oral hygiene practices and whether any other oral hygiene measures are used and distributed to the parents of a pediatric patient receiving chemotherapy. Absolute and percentage frequencies were used for statistical analysis of the data.

Results: A total of seventy-seven parents of pediatric Hematology-Oncology patients on chemotherapy completed the questionnaire. About 39\% of the parents brush their child's teeth once per day. The majority of the patients had not taken to the dentist before (75.3\%). Regarding the dental complications during chemotherapy, more than half of the patients complained of tooth pain. Most of the parents (70\%) received preventive dental advice during chemotherapy. For the mouth wash, $81.8 \%$ of the parents use mouthwash for their child. The majority of the parents (93.5\%) are interested in the dental care of their children.

Conclusion: The availability of basic oral care protocol, which includes preventive, supportive measures, and management of oral complications will increase the quality of care provided to those medically comprised patients. Also, the need for educational programs for both the patients and the guardian to increase the knowledge and awareness of the importance of oral care and daily oral hygiene practices during or after the completion of the treatment.
\end{abstract}

Keywords: Chemotherapy, pediatric, parents, oral care

\section{Introduction}

Multiple pediatric hemato-oncological disorders are treated with chemotherapy as a primary treatment approach. Chemotherapy works by inhibiting the synthesis or function of the vital nucleic acids in both cancer and healthy cells, and it is most active on cells with high growth potential including epithelial cells of the oral mucosa.[1-4]

The overall incidence of childhood cancers internationally is increasing. The annual age-specific cancer incidence rate in Saudi Arabian children is 99.83 per million, which is lower than worldwide cancer incidence. The most common reported cancers are lym-

How to cite this article: Alkhuwaiter SS. Parents' Awareness and Oral Health Care Measures of Pediatric Patients Receiving Chemotherapy. J Pediatr Dent 2021;7(1):00-00 
phoid leukemia followed by brain tumors, and then Hodgkin's lymphoma.[5] On the other hand, due to the high rate of consanguinity, the prevalence of hemoglobinopathies in Saudi Arabia is considered high (4.50\%) compared to Middle East countries (0.05\%).[6]

Hematopoietic cell transplantation preceded by intensive chemotherapy is usually the treatment of choice for relapsed oncological cases or children with genetic blood diseases.[7]

Chemotherapeutic agents can directly or indirectly have a stomatotoxicity effect on the oral and/or dental structures. [4] So, oral and dental care of the immunocompromised patient receiving chemotherapy is very important, because it will affect the course of the treatment and the quality of life of the child.[8] As well as the oral complications will have an overall adverse effect on systemic health.[9-12]

Ideally, the dental examination, treatment, and preventive measures should be carried out before the immunosuppression periods begin as a consequence of treatment with chemotherapy, including removing all potential sources of infection and advice the patient's parent of the preventive plan that will be followed during the course of treatment.[13-18]

Educating the parents of the pediatric immunocompromised patient of the importance of implementing daily basic oral care throughout the treatment is mandatory to avoid or at least reduce the incidence of acute and long-term complications.[13,16-18]

Recently the Multinational Association of Supportive Care in Cancer/International Society of Oral Oncology (MASCC/ISOO) guidelines indicated that the best way and the most important prescriptions for prevention and/or management of oral mucositis is good oral hygiene.[2,19]

Treating those patients requires a multidisciplinary team, including but not limited to pediatric oncologist/ hematologist, pediatrician, dietitian, nurses, pediatric dentist, social workers, and parents of the pediatric patient. Ensure good communication with them will improve the quality of care provided to the child. $[13,20]$

The parents of the patient with a medical condition are overprotective, and usually they are not following a strict protocol and less compliance with basic oral hygiene measures and a healthy diet. Those children also are on pediatric medications which contain sucrose and high-calorie nutritional supplements to increase the energy level.[21-23]

Moreover, the parents of those children are more concerned about the existing disease and the systemic health of their child and tend to neglect the oral care.
This could be attributed to the lack of awareness of the importance of maintaining a dental home and regular dental visits until an acute dental emergency occurs.

Following a daily basic oral care strategy will prevent the infections and subsequent bacteremia, reduce the possibility of pain in the oral and dental structures, side effect of chemotherapy on the oral soft tissue, allow good hydration through proper oral intake, reduce the length of hospital stay, and enhance the quality of life and care provided to children on chemotherapy.[13$15,17,19,23,24]$

There are very few studies published to assess the parents' awareness, knowledge, attitude, and oral health care measures of their child during chemotherapy and immunosuppression periods. One study showed that parents of pediatric cancer patients receiving chemotherapy are unaware of dental health and most of them did not take their child to the dentist previously.[25] Another study found that most of the parents of an oncological child have such knowledge on the importance of oral health, however very few are compliant with it. Also, all the parents reported that the dentist should be a member of the oncology team.[26]

Since the parents' awareness and attitude will affect the oral health of their children and will have a strong impact on basic oral hygiene practices during the chemotherapy and immunosuppression periods. This study was carried out to assess oral hygiene practices; toothbrushing frequency, whether any other oral hygiene measures are used. Dietary habits will be also assessed in the questionnaire; type of diet, sweetened beverages/snacks, and their frequency. The regularity of dental attendance or any previous prophylactic dental treatment. Also, to evaluate the parents' interest in maintaining good oral hygiene of their immunosuppressed child.

\section{Materials and Methods}

A Cross-Sectional Questionnaire-Based Study was carried to assess the parent awareness and practices towards oral health care of children receiving chemotherapy. A convenience study sample compromising seventy-seven parents of children attended at King Abdullah Specialized Children Hospital (KASCH), Department of Pediatric Hematology and Oncology.

The study was carried out between July 2020 and December 2020. Ethical approval and informed consent for carrying out the present study were obtained by the King Abdullah International Medical Research Center (KAIMRC). 
All children in the age group of 1-13 years who is

1. Suffering from cancer and receiving chemotherapy or

2. Receiving chemotherapy before bone marrow transplantation.

was included in the study.

Those who finished the chemotherapy course, patients unaccompanied by their parents at the time of the research study, those unwilling to fill in the questionnaire, and gave incomplete answers to the questionnaire were excluded from the study.

The paper-based questionnaire that was used in this study was an anonymous self-administered pretested structured questionnaire that is developed and validated by Gupta et al[25] The questionnaire was slightly modified to achieve the objectives of this study.

The questionnaire comprised of demographic information and 15 closed-ended questions assessing oral hygiene practices; toothbrushing frequency, whether any other oral hygiene measures are used. Dietary habits will be also assessed in the questionnaire; type of diet, sweetened beverages/snacks, and their frequency. The regularity of dental attendance or any previous prophylactic dental treatment will be asked.

The questionnaire was distributed to the parents of children during receiving chemotherapy or the followup visit to the physician at the outpatient clinic of the Pediatric Hematology/Oncology Department. The validity of the questionnaire was tested with $10 \%$ of the sample in a pilot study, to make sure the questionnaire is understandable, clear, and relevant.

All data were managed and statistically analyzed using the Statistical Package for the Social Sciences soft- ware (SPSS Inc., Chicago, IL, US). Absolute and percentage frequencies were obtained for qualitative variables and measures.

\section{Results}

A total of seventy-seven parents of a pediatric patient on chemotherapy completed the questionnaire. Age range from one year and up to 13 years old. The mean age was 6.8 years $(\mathrm{SD}=3.3)$. Table 1 shows the frequency of toothbrushing among hematology-oncology pediatric patients. About $39 \%$ of the parent brush their child's teeth once per day, followed by the parent who never brushes their child's teeth (33.8\%). Table 2 provides the parents' responses to oral/dental health care measures during receiving chemotherapy. The majority of the pediatric oncology patient had not taken to the dentist before $(75.3 \%)$, and $92 \%$ had no history of fluoride application. Regarding the dental complications during chemotherapy, more than half of the patients complained of tooth pain during chemotherapy, and about $58 \%$ complained of mouth sores.

About $88 \%$ of the parent was given dietary counseling by pediatrician or dietitian. However, $85.7 \%$ of the children consume sweetened beverages and/or snacks between the meals.

Table 1. Frequency of toothbrushing among hematologyoncology pediatric patients

\begin{tabular}{|l|c|c|}
\hline \multicolumn{3}{|c|}{ How many times does your child brush a day? } \\
\hline Never brush & Brush once & Brush twice or more \\
\hline $26(33.8 \%)$ & $30(39 \%)$ & $21(27.3 \%)$ \\
\hline
\end{tabular}

Table 2. Parents' responses to oral/dental health care measures of children receiving chemotherapy

\begin{tabular}{|c|c|c|}
\hline Question & Yes (\%) & No (\%) \\
\hline Whether your child's physician recommends not to brush your child's teeth during chemotherapy periods? & $15(19.5)$ & $62(80.5)$ \\
\hline Whether taken your child to a dentist before starting chemotherapy? & $19(24.7)$ & $58(75.3)$ \\
\hline History of any fluoride application? & $6(7.8)$ & $71(92.2)$ \\
\hline Has your child ever complained of tooth pain during chemotherapy periods? & $41(53.2)$ & $36(46.8)$ \\
\hline Whether referred your child to a pediatric dentist to treat emergency dental pain or abscess? & $10(13)$ & $67(87)$ \\
\hline History of bleeding in child's oral cavity? & $15(19.5)$ & $62(80.5)$ \\
\hline Whether complaints of mouth sores during chemotherapy periods? & $45(58.4)$ & $32(41.6)$ \\
\hline Whether consumes sweetened beverages or eating snacks between meals? & $66(85.7)$ & $11(14.3)$ \\
\hline Whether given dietary counseling by the pediatrician or dietitian? & $68(88.3)$ & $9(11.7)$ \\
\hline Have you received any preventive dental advice during therapy? & $54(70.1)$ & $23(29.9)$ \\
\hline Whether your child complains of dry mouth during therapy? & $33(42.9)$ & $44(57.1)$ \\
\hline Whether using mouthwash for your child? & $63(81.8)$ & $14(18.2)$ \\
\hline If yes, Does your child use the mouthwash daily? & $37(48.1)$ & $26(33.8)$ \\
\hline Are you interested in dental care for your child? & $72(93.5)$ & $5(6.5)$ \\
\hline
\end{tabular}


Most of the parents (70\%) received preventive dental advice during chemotherapy. For the mouth wash, $81.8 \%$ of the parent use mouthwash for their child. The majority of the parents of children receiving chemotherapy (93.5\%) are interested in dental care for their children.

\section{Discussion}

The present study was undertaken to assess the awareness of pediatric oncology/hematology parents regarding the oral health status and practices during chemotherapy. In this study, most of the parents (93.5\%) are interested in the dental care of their child and they are willing to maintain the health of the oral and dental structures. However, due to the severity of their child's condition, dental care will be a secondary goal to the majority of the parents and usually, they are concerned more about the existing medical condition. Similar results to this study, Gupta et al (2016) reported a high percentage of parents interested in the dental care of their child.[25]

The previous thinking of inducing bacteremia or bleeding by toothbrushing has not been established.[2730] About $39 \%$ of the parents in this study brush their child's teeth once per day, followed by $33.8 \%$ of the parents who never brush their child's teeth during the chemotherapy. In this study almost all of the parents using foam toothbrushes and not the regular toothbrush, because they think that the regular toothbrush will induce gingival bleeding. Other parents reported the foam toothbrush is enough to maintain good oral hygiene because they were given the sponge brush from the beginning of treatment with chemotherapy. On the other hand, some parents are not aware of maintaining good oral hygiene will reduce the oral side effects of chemotherapeutic agents. In contrast to the study done by Pedrosa et al (2019) to assess the parents' knowledge about the oral health care of oncological children, about $60 \%$ of oncology pediatric patients brush their teeth 3 times per day and most of them (97\%) used toothbrushe.[26]

This study revealed that more than half of the parents $(75.3 \%)$ did not take their child to the dentist before starting chemotherapy. Gupta et al (2016) reported a higher percentage of children that had not been taken to the dentist previously.[25] For hematopoietic cell transplantation patients, complete oral/dental assessment was provided more frequently compared to that in oncological cases. This may be related to the limited time between the initial cancer diagnosis and the start- ing of the treatment regimen. Also, the psychological impact of the parents of the diagnosis gives the dental appointment less priority. It could be related also to the lack of awareness of medical staff of the importance of initial oral assessment.

The American Academy of Pediatric Dentistry (AAPD) recommends pediatric oncology/hematology patients to visit the pediatric dentist for dental assessment and management before starting chemotherapy. [31] This is very important and should be included in the protocol for the management of immunocompromised pediatric patients.

The advantages to visit a pediatric dentist are; to educate the parents and the patients about how to maintain good oral hygiene during immunosuppression periods to reduce the oral side effect of chemotherapeutic agents. Inform the parents about the possible acute and long-term effects of chemotherapy on the oral cavity and the complications that might encounter during the immunosuppression.[18] Also, to establish an individualized preventive and monitoring plan to be followed during and after the course of the treatment, which include, the type of toothbrush and toothpaste, and what to use in case of severe pancytopenia and very bad oral hygiene with gingivitis.

Elad et al (2008) reported that the possibility of mortality secondary to dental infections will be reduced if the dental treatment was carried out before starting chemotherapy and subsequent immunosuppression.[14]

Verbal and written oral health care instructions that should be given to the pediatric patient's guardian include mechanical cleaning of teeth by using a soft toothbrush, two to three times per day and chemically by using fluoridated toothpaste.[19,32-34] In case of severe pancytopenia, very bad gingival health, or development of mucositis in the oral cavity, a foam brush soaked in alcohol-free chlorhexidine mouth rinse can be used temporally to decrease bacterial plaque overload.[35-37] Once the systemic and oral health improves, the regular soft toothbrush can be used for more effective dental plaque removal.[19,32] Children under 8 years of age may need to be assisted with toothbrushing and should be at least for 2 minutes. For interdental cleaning, the parents should be advised to use the devices once per day and only if they are trained enough.[32] For children who cannot swish and spit, swab the mouth rinse over dental and oral structures.

Topical professional fluoride application before or during chemotherapy is very beneficial. Fluoride has a cariostatic effect and is used to prevent, control, or arrest existing incipient or cavitated caries lesions.[38] 
Especially if the child has xerostomia as a consequence of chemotherapy. In the present study, most of the oncology/hematology patients (92\%) had no history of fluoride application. Another study indicated that all oncology pediatric patients had no history of fluoride application. [25] Many fluoride preparations are available and should be prescribed according to the age and caries risk of the individual patient. For example, fluoridated toothpaste, fluoride supplements, neutral fluoride gels/rinses, or professional application of topical fluoride.

The only recommendation that was given by the pediatric oncologist in this study is to use the mouthwash with a foam brush. Most of them prescribed (Chlorhexidine Gluconate $0.2 \%+3 \%$ Sodium Bicarbonate mouthwashes) for their patient. And in case of pain in the oral cavity or development of mucositis, mixed medications mouthwash which contains (local anesthetics, antifungal, antacid, antihistamine, and distilled water) were used. This can be explained by the limited guidelines to be followed based on the high quality of evidence for oral care protocol for pediatric patients on chemotherapy. So, the majority of parents in this study (82\%) use mouthwash for their child as being advised. But not all of them using it daily. In this study, the oral care instructions before starting the treatment were provided by the pediatric oncologist. But, for hematopoietic stem cell transplantation patients the instructions were provided by the pediatric dentist who did the dental clearance for the patient.

No strong clinical recommendations based on the high quality of evidence regarding using mixed medications mouth rinses. This is due to the heterogenicity of the studies that were used a different combination of mouthwashes among pediatric patients on chemotherapy.

Mucosal atrophy, erythema, pain, and dysgeusia, are manifestations of mucositis, which is one of the oral complications that can be experienced by the pediatric patient as a consequence of chemotherapeutic agents. [9] Other complications such as oral or dental pain, xerostomia, mucosal ulcerations, gingival bleeding, dental caries, secondary infections are also frequently observed.[13,18]

The current study indicated that about 53\% complained of tooth pain. However, only $13 \%$ of them referred to the pediatric dentist to treat emergency dental pain or abscess. This could be attributed to the severe pancytopenia during immunosuppression periods. Which will reveal a high risk of infection and bleeding following dental treatment. Most of them will be managed by pain control and ensure good hydration until the appropriate time of dental treatment. About 58.4\% of the parents in this study reported that their child has complained of mouth ulcers during therapy periods.

One study reported that nearly $43 \%$ of pediatric oncology patients were referred for urgent treatment. [26] In contrast to the study done by Gupta et al (2016) most of the children in the study group (94\%) did not complain of dental pain. [25]

Recently, the MASCC/ISOO recommended the combination of multiagent oral care protocol, including tooth brushing, flossing, and mouth wash. Also, patient education about oral care practices during the course of chemotherapy is a newly added recommendation to this guideline. Following this protocol can decrease the incidence and severity level of oral mucositis and other oral/dental complications.[37,39]

Using saline or sodium bicarbonate is based on an expert opinion, these mouth rinses are inert and bland. It will reduce the dryness and the acidity of the oral cavity. So, the patient can rinse frequently with these bland solutions. To avoid burning sensation or dryness of the oral mucosa, an alcohol-free chlorhexidine $0.12-0.2 \%$ is recommended in case of very bad oral hygiene with inflamed gingival tissues and for prevention or treatment of oral infections.[31,39] The patient should be advised to gargle, or the foam brush can be socked in chlorhexidine and to maximize its effectiveness, it should not be used with any fluoride product, keep at least 30 minutes apart. $[40,41]$ Also, due to its broad-spectrum antimicrobial, some antifungal, and antiviral action, it will suppress the pathogens in the oral cavity.[42] Chlorhexidine has been shown to reduce the duration, severity, and symptoms of mucositis, including mouth pain and difficulty in eating and swallowing.[43] A new guideline for using benzydamine mouthwash, which is an anti-inflammatory agent suggested for the prevention of oral mucositis in patients with head and neck cancer receiving radiotherapy and chemotherapy.[37,39]

Da Fonseca (2004) reported that, by the implementation of intensive oral care, the incidence and the complications of mucositis have been reduced.[44] So, the best way to prevent oral complications during the course of treatment is to maintain good oral hygiene through daily basic oral care.

Include a written leaflet to the verbal advice in an easy and clear language about oral care practices given to the patients and parents may improve the compliance. Also, it will increase the parent knowledge towards oral health of their child.[45]

The dietary counseling of a patient undergoing chemotherapy is usually given by the dietitian. Ensuring a proper nutritional status through good hydration and a 
balanced healthy diet is important for those patients. The majority of the parents in this study were given dietary counseling. On the other hand, about $86 \%$ of the parents reported that their child consumes sweetened beverages or eating snacks between the meals. A possible explanation of this is related to the behavior of the parents with their medically compromised child. Usually, they will be overprotective and overindulgent, and less strict with the favorite food of their child.

Those patients are considered to be at high risk for dental caries according to the AAPD caries risk assessment tool. Moreover, previous studies reported that the prevalence of carious lesions in primary teeth is higher significantly in cancer patients receiving chemotherapy compared to a healthy patient. [46,47]

The increased caries risk in this group of a pediatric patient may be related to the high-calorie, high-protein food contents in a daily supplement, the high amount of sucrose in the pediatric medications, and the increased consumption of sweetened beverages and snacks between the meals. $[18,22,41]$ So, communication with the patient's dietitian is highly recommended to ensure that the nutritional supplements do not outweigh the highly cariogenic diet.

In a survey that was conducted on 22 centers in the UK regarding the oral care practices of pediatric cancer patients, there was variation between the centers in prevention and intervention measures. On the other hand, there was an agreement in most centers on routine basic oral hygiene which include, brushing with a soft toothbrush two times per day. Of 22 centers, only 8 (36\%) reported that children visited the dentist before starting chemotherapy.[48] This is similar to the survey among health care providers, there was a wide variation in the recommendations for clinical practices of prevention and management of oral side effects among cancer patients.[49]

\section{Conclusion}

The need for internationally accepted evidence-based clinical practice guidelines is highly recommended. Good communication between medical and dental providers along with the availability of basic oral care protocol, which includes preventive, supportive measures, and management of oral complications will increase the quality of care provided to these medically comprised patients. So, the medical, nursing, patient, and parents can follow these guidelines easily and efficiently. Also, the need for educational programs for both the patient and the guardian to increase the knowledge and aware- ness of oral care before, during, and after the completion of the treatment. Educational programs for medical and nursing staff also are very important, because they will be the first to contact with the patient and his/her parent. By increasing the awareness of the parents towards basic oral care during chemotherapy, they will be more compliant with the instructions given.

\section{Financial Disclosure: Nil.}

\section{Conflict of Interest: None declared.}

Funding: The author received no financial support for the research, authorship, and/or publication of this article.

Institutional Review Board Statement: The study was conducted according to the guidelines of King Abdullah International Medical Research Center (KAIMRC) and approved by the Institutional Review Board of King Abdullah International Medical Research Center (KAIMRC) (RC20/126/R, May 2020).

Informed Consent Statement: Informed consent was obtained from all subjects involved in the study.

\section{References}

1. Tarbell NJ, Kooy HM. General principles of radiation oncology. In: Pizzo PA, Poplack DG, editors. Principles and practice of pediatric oncology, 4th ed. Philadelphia: Lippincott Williams \& Wilkins; 2002. p. 369-380.

2. Lalla RV, Bowen J, Barasch A, et al. MASCC/ISOO clinical practice guidelines for the management of mucositis secondary to cancer therapy [published correction appears in Cancer. 2015 Apr 15;121(8):1339]. Cancer 2014;120(10):1453-1461 doi: $10.1002 /$ cncr. 28592

3. Miller MM, Donald DV, Hagemann TM. Prevention and treatment of oral mucositis in children with cancer. J Pediatr Pharmacol Ther 2012;17(4):340-350 doi:10.5863/1551-6776-17.4.340

4. Miller M, Kearney N. Oral care for patients with cancer: a review of the literature. Cancer Nurs 2001;24(4):241-254 doi:10.1097/00002820-200108000-00001

5. Belgaumi AF, Pathan GQ, Siddiqui K, et al. Incidence, clinical distribution, and patient characteristics of childhood cancer in Saudi Arabia: A population-based analysis. Pediatr Blood Cancer 2019;66(6):e27684 doi:10.1002/pbc.27684

6. Alsaeed ES, Farhat GN, Assiri AM, et al. Distribution of hemoglobinopathy disorders in Saudi Arabia based on data from the premarital screening and genetic counseling program, 20112015. J Epidemiol Glob Health 2018;7 Suppl 1(Suppl 1):S41-S47 doi:10.1016/j.jegh.2017.12.001

7. Majhail NS, Farnia SH, Carpenter PA, et al. Indications for autologous and allogeneic hematopoietic cell transplantation: guidelines from the American society for blood and marrow transplantation. Biol Blood Marrow Transplant 2015;21(11):1863-1869 doi:10.1016/j.bbmt.2015.07.032

8. Gandhi K, Datta G, Ahuja S, Saxena T, G Datta A. Prevalence of oral complications occurring in a population of pediatric cancer patients receiving chemotherapy. Int J Clin Pediatr Dent 2017;10(2):166-171 doi:10.5005/iD-iournals-10005-1428 
9. Sonis ST, Oster G, Fuchs H, et al. Oral mucositis and the clinical and economic outcomes of hematopoietic stem-cell transplantation. J Clin Oncol 2001;19(8):2201-2205 doi:10.1200/JCO.2001.19.8.2201

10. Vera-Llonch M, Oster G, Ford CM, Lu J, Sonis S. Oral mucositis and outcomes of allogeneic hematopoietic stem-cell transplantation in patients with hematologic malignancies. Support Care Cancer 2007;15(5):491-496 doi:10.1007/s00520-006-0176-9

11. Elting LS, Cooksley C, Chambers M, Cantor SB, Manzullo E, Rubenstein EB. The burdens of cancer therapy. Clinical and economic outcomes of chemotherapy-induced mucositis. Cancer 2003;98(7):1531-1539 doi:10.1002/cncr.11671

12. Kashiwazaki H, Matsushita T, Sugita J, et al. Professional oral health care reduces oral mucositis and febrile neutropenia in patients treated with allogeneic bone marrow transplantation. Support Care Cancer 2012;20(2):367-373 doi:10.1007/s00520011-1116-x

13. National Cancer Institute. (2002-2016). PDQ Oral Complications of Chemotherapy and Head/Neck Radiation. Bethesda, Md.: National Cancer Institute. Available at: https://www.cancer.gov/ about-cancer/treatment/side-effects/mouth-throat/oral-complications-hp-pdq. Accessed February 7, 2021

14. Elad S, Thierer T, Bitan M, Shapira MY, Meyerowitz C. A decision analysis: the dental management of patients prior to hematology cytotoxic therapy or hematopoietic stem cell transplantation. Oral Oncol 2008;44(1):37-42 doi:10.1016/j.oraloncology.2006.12.006

15. Hong $\mathrm{CH}$, Brennan MT, Lockhart PB. Incidence of acute oral sequelae in pediatric patients undergoing chemotherapy. Pediatr Dent 2009;31(5):420-425

16. Kumar N, Brooke A, Burke M, John R, O’Donnell A, Soldani F. The Oral management of oncology patients requiring radiotherapy, chemotherapy and/or bone marrow transplantation. Clinical Guidelines. The Royal College of Surgeons of England/ The British Society for Disability and Oral Health. Fac Dent J 2013;4:200-203 doi:10.1308/204268513X13776914744952

17. Lalla RV, Brennan MT, Schubert MM. Oral complications of cancer therapy. In: Yagiela JA, Dowd FJ, Johnson BS, Marrioti AJ, Neidle EA, editors. Pharmacology and Therapeutics for Dentistry, 6th ed. St. Louis, Mo.: Mosby-Elsevier; 2011. p. 782-798

18. Hong $\mathrm{CH}$, da Fonseca M. Considerations in the pediatric population with cancer. Dent Clin North Am 2008;52(1):155-ix doi:10.1016/j.cden.2007.10.001

19. Peterson DE, Boers-Doets CB, Bensadoun RJ, Herrstedt J; ESMO Guidelines Committee. Management of oral and gastrointestinal mucosal injury: ESMO Clinical Practice Guidelines for diagnosis, treatment, and follow-up. Ann Oncol 2015;26 Suppl 5:v139-v151 doi:10.1093/annonc/mdv202

20. National Institute of Dental and Craniofacial Research. (2016). Dental management of the organ or stem cell transplant patient. Bethesda, Md.: National Institute of Dental and Craniofacial Research. Available at: https://www.nidcr.nih.gov/sites/default/ files/2017-09/organ-stem-cell-transplant-mouth.pdf. Accessed January18, 2021

21. Van Dongen-Melman JE, Sanders-Woudstra JA. Psychosocial aspects of childhood cancer: a review of the literature [pub- lished correction appears in J Child Psychol Psychiatry 1986 Sep;27(5):713]. J Child Psychol Psychiatry 1986;27(2):145-180

22. Padmini C, Bai KY. Oral and dental considerations in pediatric leukemic patient. ISRN Hematol 2014;2014:895721 doi:10.1155/2014/895721

23. Hong $\mathrm{CH}$, Napeñas JJ, Hodgson $\mathrm{BD}$, et al. A systematic review of dental disease in patients undergoing cancer therapy. Support Care Cancer 2010;18(8):1007-1021 doi:10.1007/s00520-0100873-2

24. Little JW, Miller CS, Rhodus NL. Cancer and oral care of patients with cancer. In Little and Falace's Dental Management of the Medically Compromised Patient, 9th ed. St. Louis, Mo.: Elsevier; 2018. p. 501-513

25. Gupta A, Marwaha M, Bansal K, Sachdeva A, Gupta A. Dental awareness among parents and oral health of paediatric cancer patients receiving chemotherapy. J Clin Diagn Res 2016;10(5):ZC92-ZC95 doi:10.7860/JCDR/2016/17412.7819

26. Pedrosa BRV, Martins WLL, Almeida HCR, Kozmhinsky VMR, Sabino MFPA, Oliveira KMM. Parents' knowledge about the oral health care of oncological children. RGO, Rev Gaúch Odontol 2019;67:e2019008 doi:10.1590/1981-86372019000083605

27. Kubota K, Kobayashi W, Sakaki H, et al. Professional oral health care reduces oral mucositis pain in patients treated by superselective intra-arterial chemotherapy concurrent with radiotherapy for oral cancer. Support Care Cancer 2015;23(11):3323-3329 doi:10.1007/s00520-015-2774-x

28. Wong HM. Oral complications and management strategies for patients undergoing cancer therapy. ScientificWorldJournal 2014;2014:581795 doi:10.1155/2014/581795

29. Levin L, Bilder L, Borisov O. Improving oral hygiene skills among children undergoing treatment at the haemato-oncology department - an interventional programme. Int Dent J 2015;65(4):211215 doi:10.1111/idj.12171

30. Lopes NN, Petrilli AS, Caran EM, França CM, Chilvarquer I, Lederman H. Dental abnormalities in children submitted to antineoplastic therapy. J Dent Child (Chic) 2006;73(3):140-145

31. American Academy of Pediatric Dentistry. Dental management of pediatric patients receiving immunosuppressive therapy and/or radiation therapy. The Reference Manual of Pediatric Dentistry. Chicago, III.: American Academy of Pediatric Dentistry 2020;453-461

32. Schubert MM, Peterson DE. Oral complications of hematopoietic cell transplantation. In: Appelbaum RF, Forman SJ, Negrin RS, Blume KG, editors. Thomas' Hematopoietic Cell Transplantation: Stem Cell Transplantation, 4th ed. Oxford, Wiley-Blackwell, UK; 2009. p. $1589-607$

33. Qutob AF, Allen G, Gue S, Revesz T, Logan RM, Keefe D. Implementation of a hospital oral care protocol and recording of oral mucositis in children receiving cancer treatment: a retrospective and a prospective study. Support Care Cancer 2013;21(4):1113-1120 doi:10.1007/s00520-012-1633-2

34. Newbrun E. Topical fluorides in caries prevention and management: a North American perspective. J Dent Educ 2001;65(10):1078-1083 
35. Ransier A, Epstein JB, Lunn R, Spinelli J. A combined analysis of a toothbrush, foam brush, and a chlorhexidine-soaked foam brush in maintaining oral hygiene. Cancer Nurs 1995;18(5):393396

36. Epstein J, Ransier A, Lunn R, Spinelli J. Enhancing the effect of oral hygiene with the use of a foam brush with chlorhexidine. Oral Surg Oral Med Oral Pathol 1994;77(3):242-247 doi:10.1016/0030-4220(94)90292-5

37. Hong CHL, Gueiros LA, Fulton JS, et al. Systematic review of basic oral care for the management of oral mucositis in cancer patients and clinical practice guidelines. Support Care Cancer 2019;27(10):3949-3967 doi:10.1007/s00520-019-04848-4

38. American Academy of Pediatric Dentistry. Fluoride therapy. The Reference Manual of Pediatric Dentistry. Chicago, III: American Academy of Pediatric Dentistry 2020;288-291

39. Elad S, Cheng KKF, Lalla RV, et al. MASCC/ISOO clinical practice guidelines for the management of mucositis secondary to cancer therapy. Cancer 2020;126(19):4423-4431 doi:10.1002/ cncr.33100

40. Scottish Dental Clinical Effectiveness Programme. Drug Prescribing for Dentistry - Dental Clinical Guidance. 2016, Third Edition. Available at: http://www.sdcep.org.uk/wp-content/uploads/2016/03/SDCEP-Drug-Prescribing-for-Dentistry3rd-edition.pdf. Accessed December 12, 2020

41. Moursi AM, Fernandez JB, Daronch M, Zee L, Jones CL. Nutrition and oral health considerations in children with special health care needs: implications for oral health care providers. Pediatr Dent 2010;32(4):333-342
42. Gjermo P. Chlorhexidine and related compounds. J Dent Res 1989;68:1602-1608

43. Costa EM, Fernandes MZ, Quinder LB, de Souza LB, Pinto LP. Evaluation of an oral preventive protocol in children with acute lymphoblastic leukemia. Pesqui Odontol Bras 2003;17(2):147150 doi:10.1590/s1517-74912003000200009

44. da Fonseca MA. Dental care of the pediatric cancer patient. Pediatr Dent 2004;26(1):53-57

45. Semple CJ, McGowan B. Need for appropriate written information for patients, with particular reference to head and neck cancer. J Clin Nurs 2002;11(5):585-593 doi:10.1046/j.13652702.2002.00641.x

46. Cubukcu CE, Sevinir B. Dental health indices of long-term childhood cancer survivors who had oral supervision during treatment: a case-control study. Pediatr Hematol Oncol 2008;25(7):638-646 doi:10.1080/08880010802237849

47. Nemeth O, Kivovics M, Pinke I, Marton K, Kivovics P, Garami M. Late effects of multiagent chemotherapy on salivary secretion in children cancer survivors. J Am Coll Nutr 2014;33(3):186-191 doi:10.1080/07315724.2013.834802

48. Glenny AM, Gibson F, Auld E, et al. A survey of current practice with regard to oral care for children being treated for cancer. Eur J Cancer 2004;40(8):1217-1224 doi:10.1016/j.ejca.2004.01.030

49. Barker GJ, Epstein JB, Williams KB, Gorsky M, Raber-Durlacher JE. Current practice and knowledge of oral care for cancer patients: a survey of supportive health care providers. Support Care Cancer 2005;13(1):32-41 doi:10.1007/s00520-004-0691-5 